\title{
Four-quadrant linear-assisted DC/DC voltage regulator
}

\author{
Herminio Martínez-García ${ }^{1} \cdot$ Alireza Saberkari $^{2}$
}

Received: 2 December 2015/Revised: 14 March 2016/ Accepted: 15 April 2016

(C) Springer Science+Business Media New York 2016

\begin{abstract}
This Mixed Signal Letter presents a proposal of four-quadrant linear-assisted DC/DC voltage regulator. In this topology, a class-AB linear voltage amplifier assists a four-quadrant switching DC/DC converter in order to obtain a compact circuit with advantages of both alternatives; i.e., high efficiency, inherent in switching converters, and low output ripple and fast reaction to the load changes that are characteristics of linear regulators. In order to reduce the power dissipation in the linear regulator, it is considered as an assisting circuit for providing just a small fraction of the total load current. Furthermore, this stage provides the required clock signal for the switching counterpart, obtaining a compact topology thanks to the reduction of the complexity in the design of the control scheme for the switching converter. In fact, the proposed topology can be addressed to on-chip practical implementations, since no output capacitors are required. This last advantage provides the possibility of obtaining good-performance power-supply modulators for envelope tracking techniques in power amplifiers. The implementation and results indicate that the proposed four-quadrant linear-assisted DC/DC regulator can achieve a notably compacting and higher performance, while consuming less power in comparison to linear alternatives.
\end{abstract}

Herminio Martínez-García

herminio.martinez@upc.edu

1 Department of Electronic Engineering, Barcelona College of Industrial Engineering (EUETIB), Technical University of Catalonia - BarcelonaTech (UPC), C/ Comte d'Urgell, 187, E-08036 Barcelona, Spain

2 Department of Electrical Engineering, Faculty of Engineering, University of Guilan, 41635-3756, Rasht 41996-13769, Iran
Keywords DC-DC switching converters · Linear voltage regulators · Four-quadrant DC/DC switching converter . Linear-assisted DC/DC regulator · Hysteretic control

\section{Introduction}

Nowadays, the emergence of high bandwidth communication architectures involving radio frequency (RF) or audio power amplifiers (PAs), have created new challenges for powering telecommunication networks [1]. One of the most demanding functions, from a energy management perspective, is the generation of suitable power supply rails in order to supply these PAs not only with suitable positive, but also negative voltages. The PA, which is usually responsible for most of the power consumption in the entire transceiver, has poor energy efficiency, especially when transmitting low power levels [2,3]. The stringent linearity requirements in broadband systems make linear PAs, particularly class A, the preferred choice in the transceiver [4]. This linearity requirement, coupled with the fact that the PA operates at low transmit power during most of its operation, makes the power efficiency of the entire transceiver poor. Consequently, wireless transmitters usually employ envelope-tracking techniques for the power-supply subsystem, and they are becoming popular nowadays since they provide significant power savings [5]. In these systems, the supply modulator, usually a switching regulator in order to achieve good efficiency generates the PA supply voltage while its output tracks the signal envelope. The aforementioned conventional switching regulators are usually efficient, but have slow response; since switching frequencies cannot be drastically increased.

A solution to enhance the transient response of a switching converter consists of a combination of a linear 
regulator (or class-AB amplifier) in parallel with a switching one. This architecture is referred as linear-assisted hybrid regulator or master-slave modulator. This one-quadrant structure increases the response speed of the supply modulator while maintaining other performances, such as small ripple and small switching losses [6]. However, achieving four-quadrant linear-assisted DC/DC regulator usually involves increasing the complexity of the power supply modulator.

In this Mixed Signal Letter, the proposal of a four-quadrant DC/DC linear-assisted switching regulator is presented. A class-AB linear amplifier, together with a suitable control, assists a four-quadrant DC/DC switching converter that uses only two switches, simplifying the architecture. Limiting the maximum value of the current flowing through the linear amplifier, high efficiency can be achieved, whereas high output capacitors, normally bulky and with poor frequency response, can be removed from the circuit.

In addition, this four-quadrant proposal can be a candidate not only in the power supply path of the supply modulator, but also in the signal path (with a suitable component design according to the frequency requirements) in order to overcome the major drawbacks associated to audio and RF class-D power amplifiers. They are widely used in consumer portable electronic devices and industrial applications, such as mobile phones and laptops audio amplifiers, etc., thanks to their high efficiency. Nevertheless, notice that these amplifiers have a limited linearity due to their switching behavior and a limited control bandwidth [7-9]. Therefore, the use of a four-quadrant linear-assisted amplifier (also named as class-AD amplifier) can avoid these restrictions associated to class-D amplifiers.

This Mixed Signal Letter is organized as follows: Sect. 2 reviews the four-quadrant DC/DC switching converter that uses only two switches. In addition, Sect. 3 introduces the proposal of a four-quadrant DC/DC linearassisted switching regulator. On the other hand, as a proof of concept, Sect. 4 provides simulation and experimental results in order to corroborate the proposed design presented in previous section. These results are obtained for both low-frequency (audio class-AD power amplifier) and high-frequency applications (CDMA IS-95 specifications and WCDMA). Finally, the main conclusions obtained in the article are presented in Sect. 5.

\section{Double-switch four-quadrant DC/DC switching converter}

Figure 1 shows the basic structure of the four-quadrant converter [10]. Considering the basic operation, an nMOS transistor, $M_{1}$, and a pMOS one, $M_{2}$, operate in opposite phase and at a constant switching frequency. Notice that some control techniques can be used (not shown in the

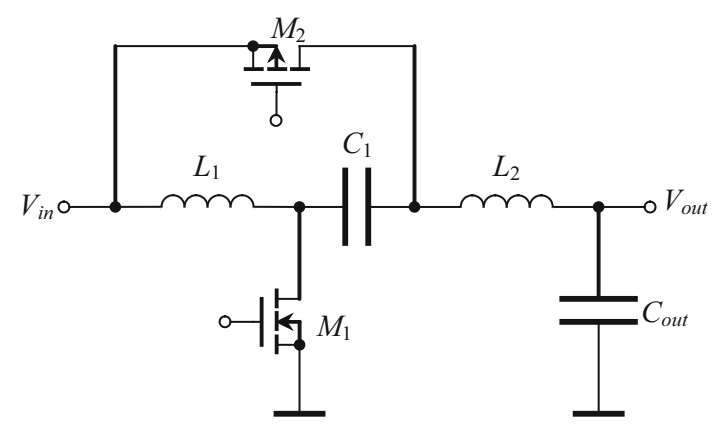

Fig. 1 Basic topology of the four-quadrant DC/DC switching converter

figure) in order to obtain output voltage regulation (for instance, current mode control to modulate the duty cycle of the transistors, as needed). By considering the fixed frequency operation, conversion ratio, $M$, of the switching converter can be obtained as:

$M=\frac{V_{\text {out }}}{V_{\text {in }}}=\frac{2 D-1}{D-1}$,

in which $D$ is the duty cycle for the on time of the transistor $M_{2}$. As it can be seen, with a positive input voltage, $V_{i n}$, the output voltage, $V_{\text {out }}$, is positive (up to the input voltage $V_{i n}$ ) for $0 \leq D<0.5$, or negative for $0.5<D<1$, limited by practical duty cycle considerations in order not to fall into instability conditions. In addition, note that for $D=0.5$, output voltage $V_{\text {out }}$ can reach zero as well. Furthermore, the output of the converter can sink or source current regardless of the polarity of the output voltage, providing a true four-quadrant operating conditions [10].

\section{Proposal of a four-quadrant DC/DC linear- assisted switching regulator}

This Mixed Signal Letter shows the proposal of including a linear amplifier, together with a suitable control, to assist the aforementioned four-quadrant DC/DC switching converter. A simple way to control this four-quadrant DC/DC converter consists of using a linear voltage regulator, which is able to assist the switching converter, obtaining a fourquadrant linear-assisted DC/DC voltage regulator. Figure 2 shows the block diagram of the basic structure of the proposed four-quadrant DC/DC linear-assisted switching regulator. Thanks to this technique, in addition to having a simple control technique, the output capacitor, normally with high value, bulky, and with poor frequency response (since it should be electrolytic), can be removed from the circuit. Thus, it is important to highlight that the linear voltage regulator acts as an "active output capacitor" in order to filter output voltage ripples. 


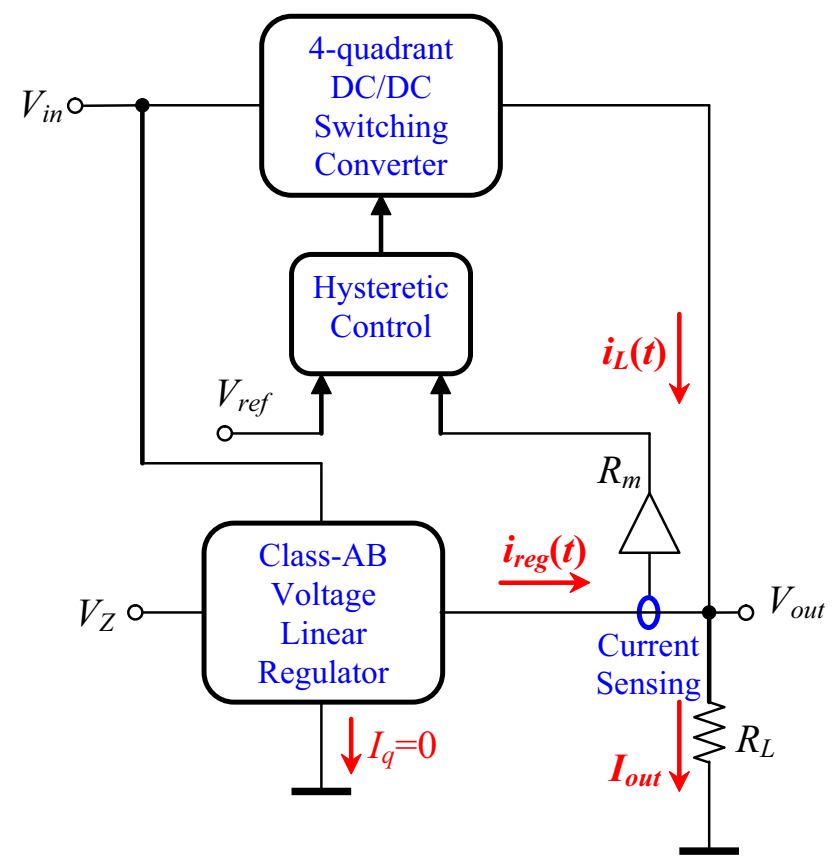

Fig. 2 Block diagram of the basic structure of the proposed fourquadrant DC/DC linear-assisted switching regulator

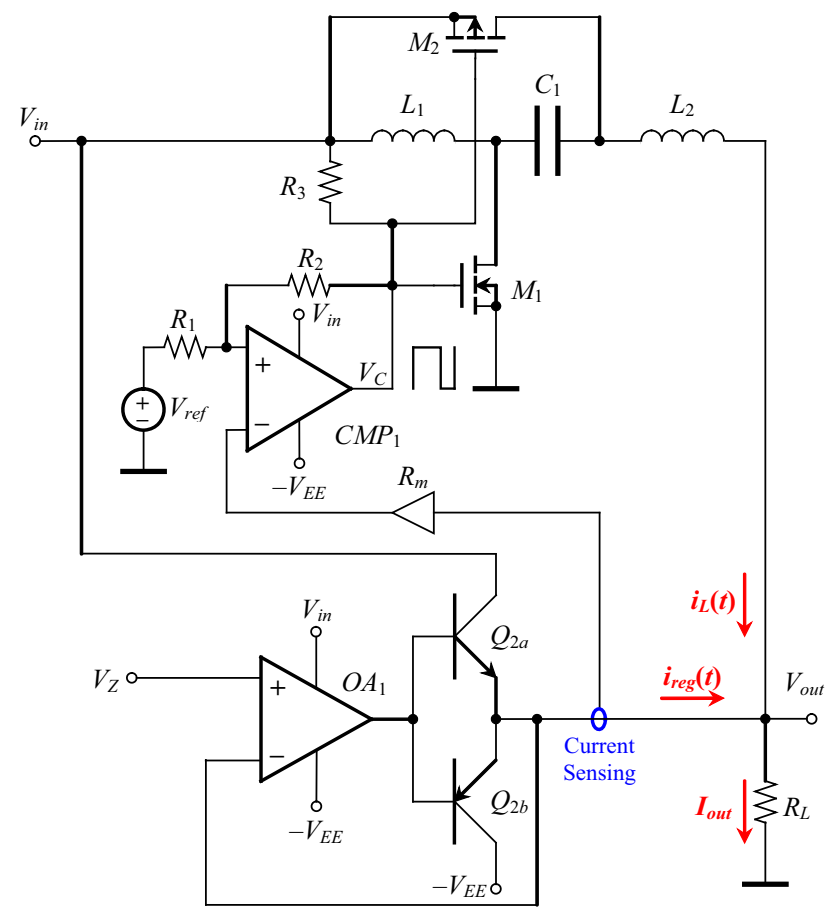

Fig. 3 Circuit implementation of the four-quadrant DC/DC linearassisted switching regulator

Figure 3 shows the circuit implementation of the proposed four-quadrant linear-assisted switching regulator. The circuit consists of a linear voltage regulator that assists the four-quadrant DC/DC switching converter presented in
Fig. 1. The control of this converter is carried out by sensing the current flowing through the linear regulator $i_{\text {reg }}(t)$ and using a hysteretic controller based on an analog comparator $\left(C M P_{1}\right)$ that controls MOS transistors $M_{1}$ and $M_{2}$. As commented before, these transistors operate out of phase from each other. However, unlike the basic fourquadrant DC/DC switching converter, in this case, the linear-assisted version works at a variable switching frequency due to the aforementioned hysteretic controller.

Obviously, if the reference voltage, $V_{Z}$, at the input terminal of the linear regulator is constant, the output voltage, $V_{\text {out }}$, will also remain constant. Thus, the output current provided by the circuit will be also constant and regulated, independently of load and/or input voltage variations, achieving good output voltage regulation without any need to include the output capacitor $C_{\text {out }}$ (which is shown in Fig. 1) [11].

To maintain the efficiency at the same level of a sole switching converter, the linear regulator is considered as an assisting circuit for providing just a small fraction of the load current while the excess current is supported by the four-quadrant switching converter. Consequently, the output current can be written as:

$I_{\text {out }}=i_{\text {reg }}(t)+i_{L}(t)$,

The aforementioned analog comparator $C M P_{1}$ determines the control of the switching converter. If the load current is below a boundary current value, named threshold switching current, $I_{\gamma}$, the comparator $C M P_{1}$ disables the switching converter. Consequently, the current through the inductance $L_{2}$ will be zero. Therefore, the linear regulator supplies the load $R_{L}$, providing all the output current $\left(I_{\text {reg }}=I_{\text {out }}\right)$.

Both the reference voltage, $V_{r e f}$, and sensor's transresistance, $R_{m}$, fix the value of a threshold switching current $I_{\gamma}$, according to:

$I_{\gamma}=\frac{V_{r e f}}{R_{m}}$

Notice that this threshold switching current, is the maximum value of the average current flowing through the linear regulator block at the steady state, as we can appreciate in Fig. 4. Thus, if the output current overtakes this value, the switching DC/DC converter will provide the difference. It is noted that, selecting an appropriate $I_{\gamma}$ (with the objective of not decreasing the efficiency), the circuit has the ability to provide almost all the output current $I_{\text {out }}$ thanks to the switching converter. In fact, under these conditions, only a small fraction of the aforementioned output current is provided by the linear regulator, which acts as an "active output capacitor" in order to filter output voltage ripples.

In addition, when the reference voltage, $V_{Z}$, at the input terminal of the linear regulator is variable, adopting 
Fig. 4 Transient response of the output load current $\left(I_{\text {out }}\right)$, switching DC/DC converter's output current $\left(i_{L}(t)\right)$, and linear voltage regulator' current $\left(i_{\text {reg }}(t)\right)$

Fig. 5 Currents flowing through the load resistor $\left(i_{\text {out }}(t)\right)$, inductance $L_{1}\left(i_{L}(t)\right)$, and linear regulator $\left(i_{\text {reg }}(t)\right)$ at the steady state when the input voltage $V_{Z}$ is a single $4-\mathrm{V}$ $20-\mathrm{kHz}$ tone, and the linearassisted switching regulator provides a peak current of $4.0 \mathrm{~A}$ to the $1-\Omega$ output load

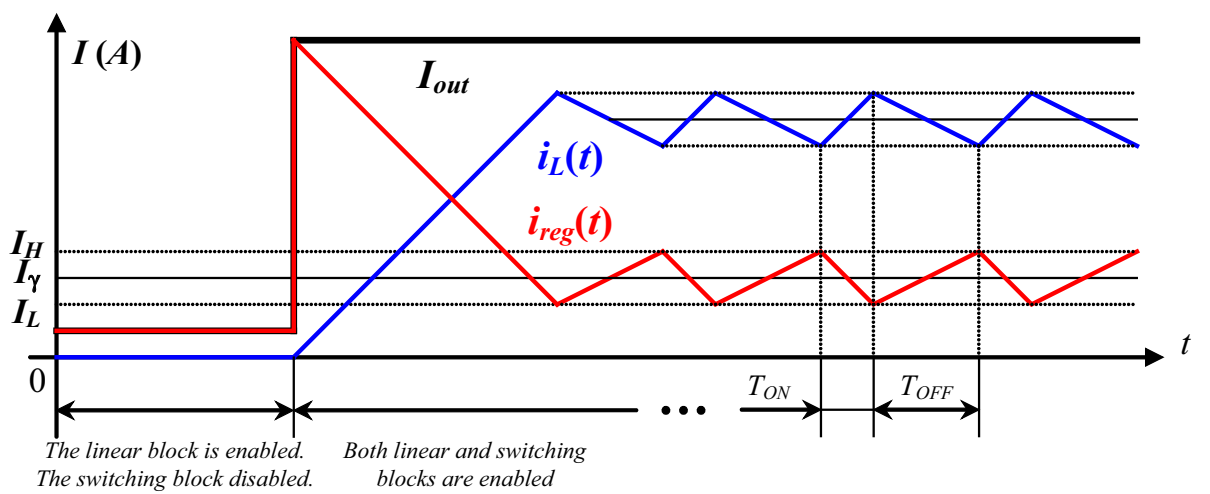

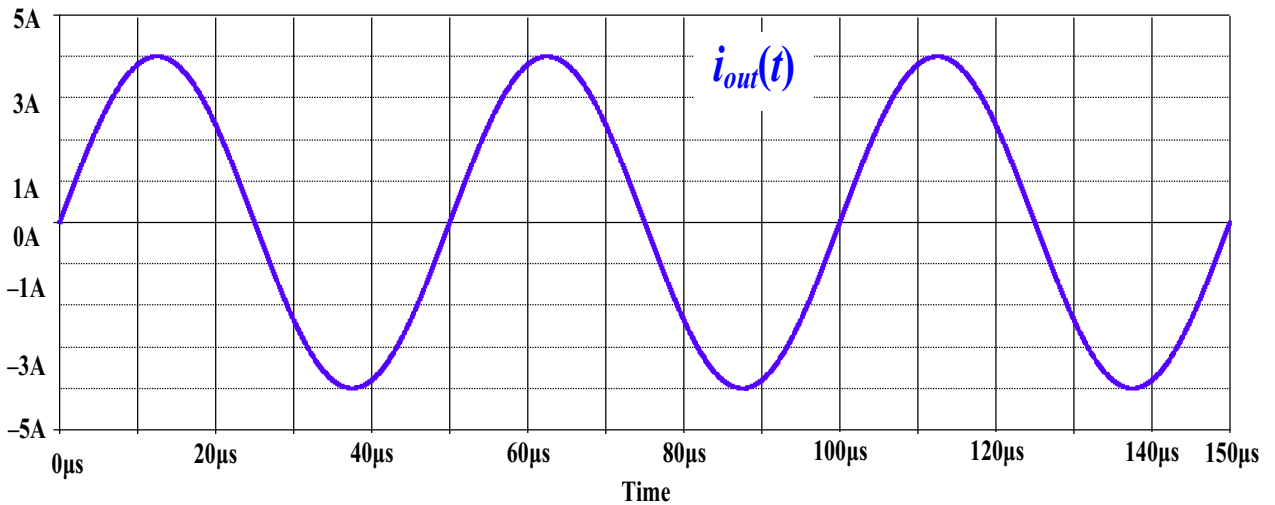
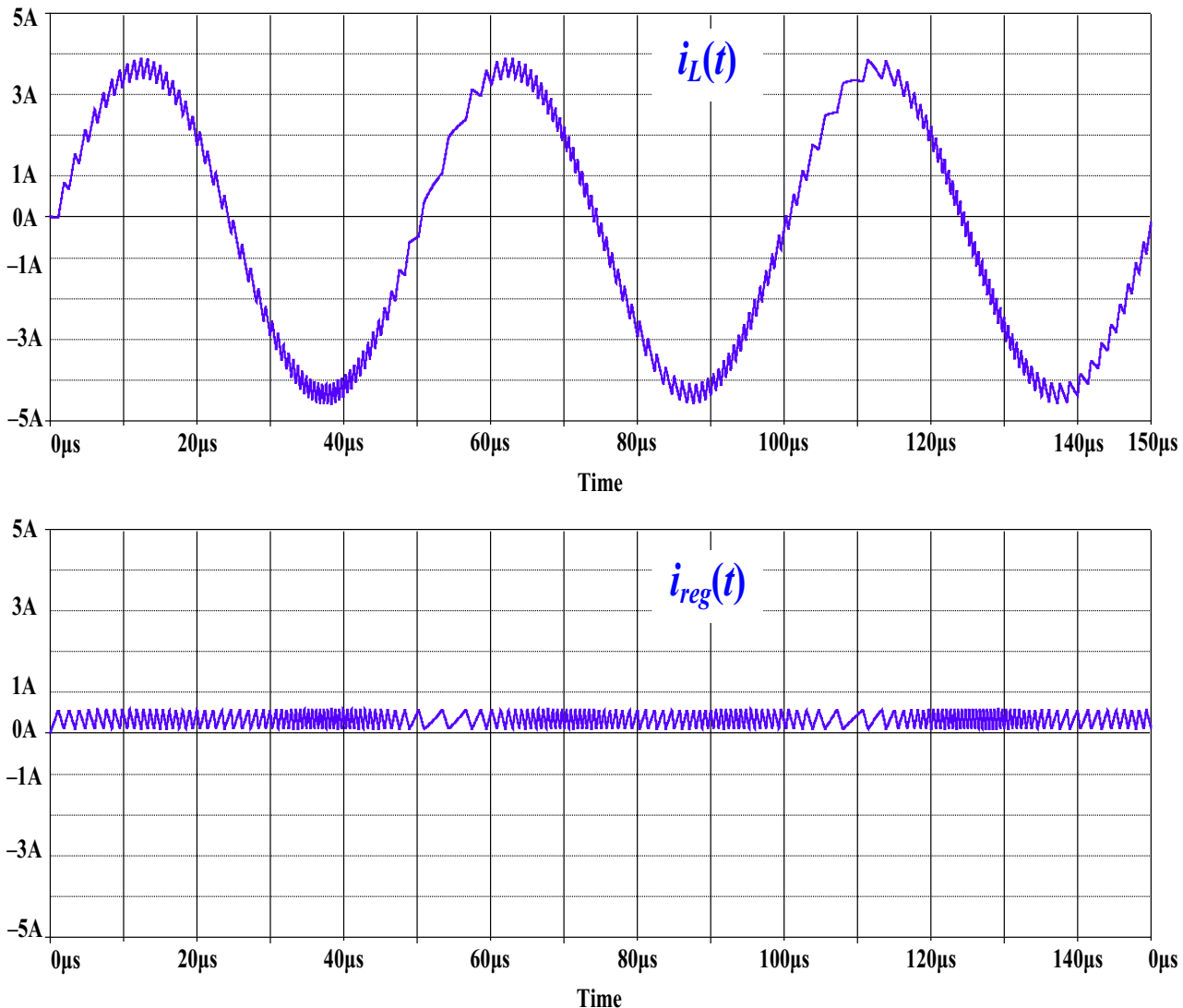
Fig. 6 Comparison of efficiencies of the four-quadrant DC/DC switching converter (blue line), the proposed fourquadrant DC/DC linear-assisted regulator (red line), and the linear regulator (pink line), with respect to variation in the output current (Color figure online)
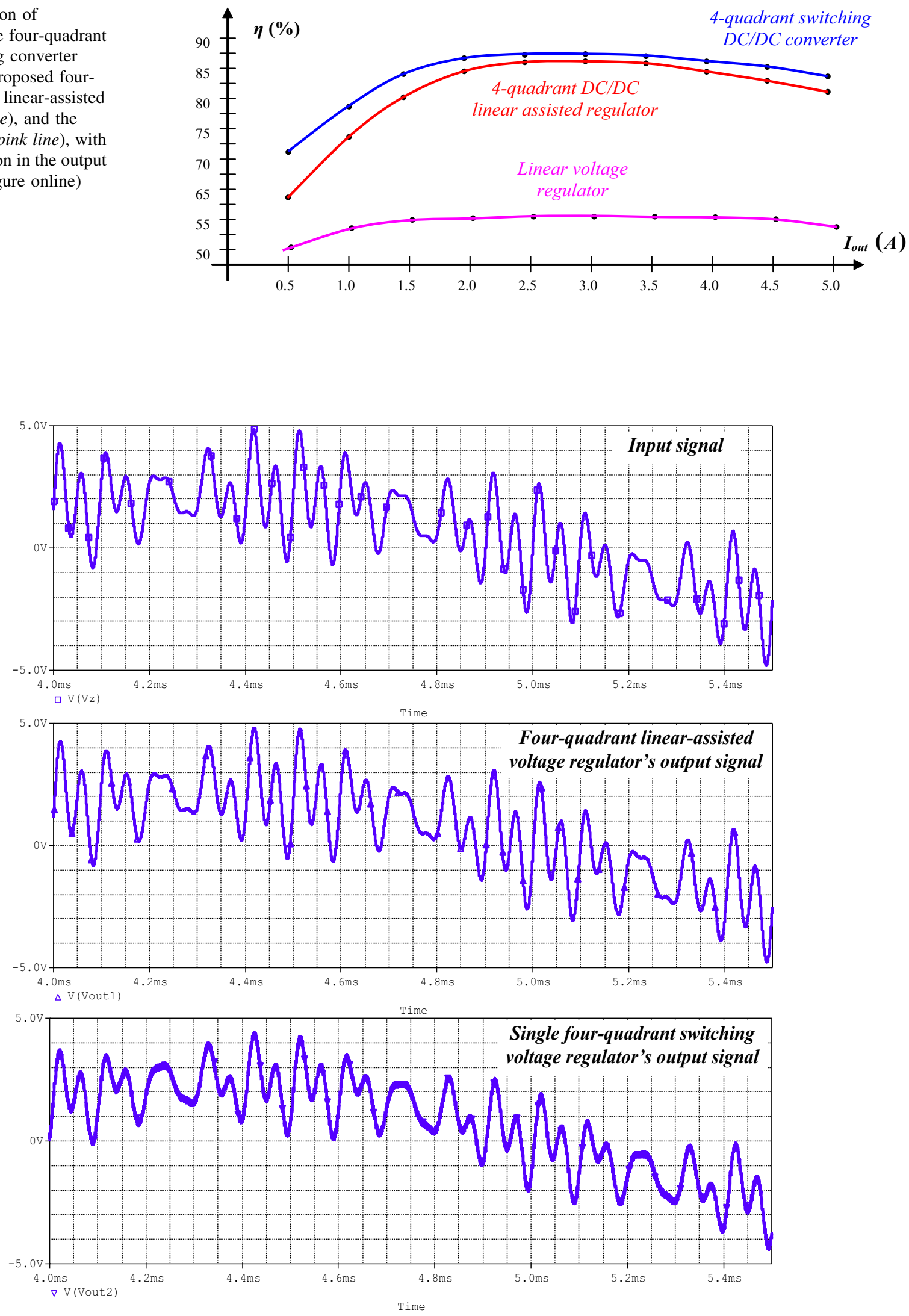

Fig. 7 Input and output signals of proposed four-quadrant linear-assisted voltage regulator, and the single four-quadrant switching voltage regulator with a multi-tone time-varying audio input signal as a candidate for class-AD audio power amplifier 
Fig. 8 Transient response for CDMA IS-95 specifications: Input and output signals of proposed four-quadrant linearassisted voltage modulator, and the single four-quadrant switching voltage modulator to observe the dynamic converter's response to a worst case power adjustment from $26 \mathrm{dBm}$ $(2.95 \mathrm{~V})$ to $27 \mathrm{dBm}(3.6 \mathrm{~V})$

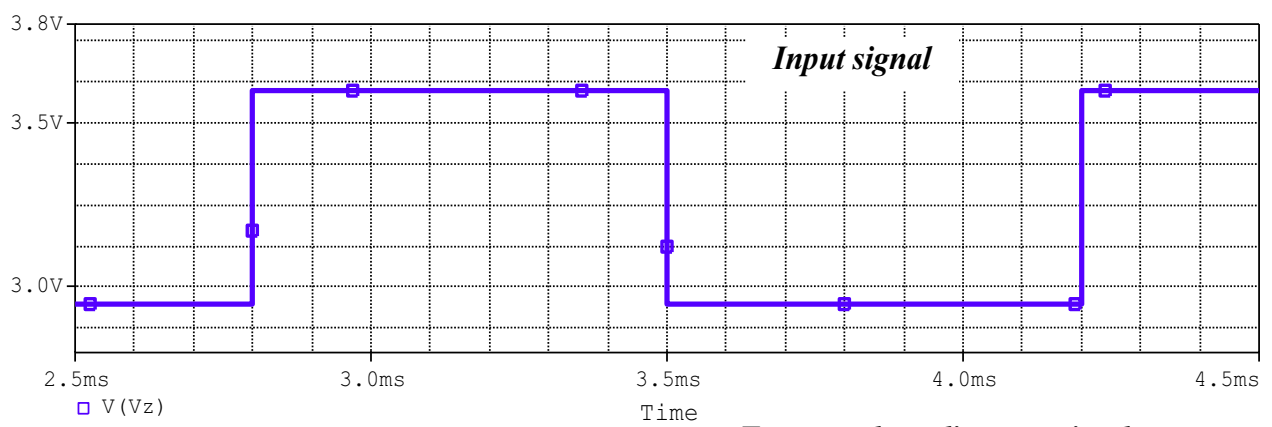

Four-quadrant linear-assisted

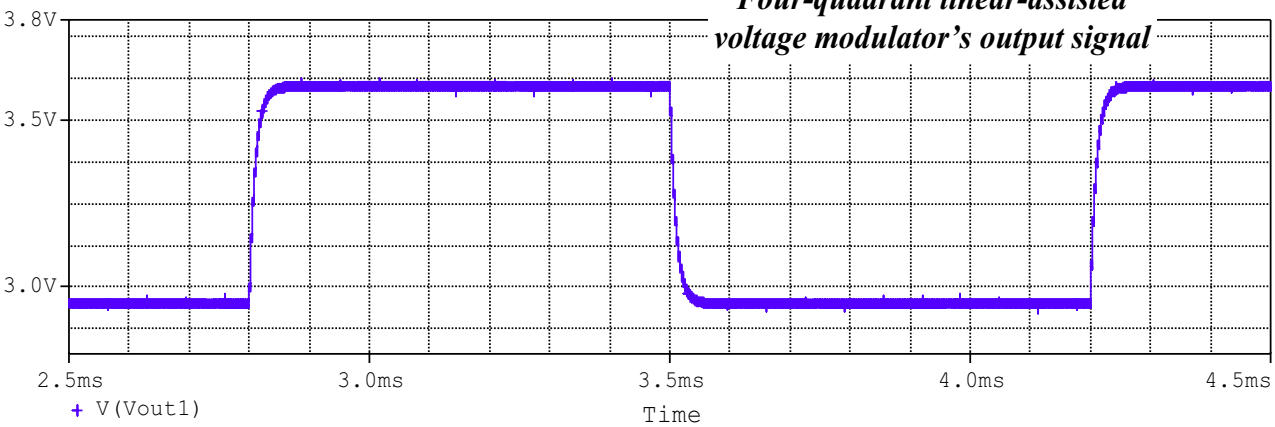

Time

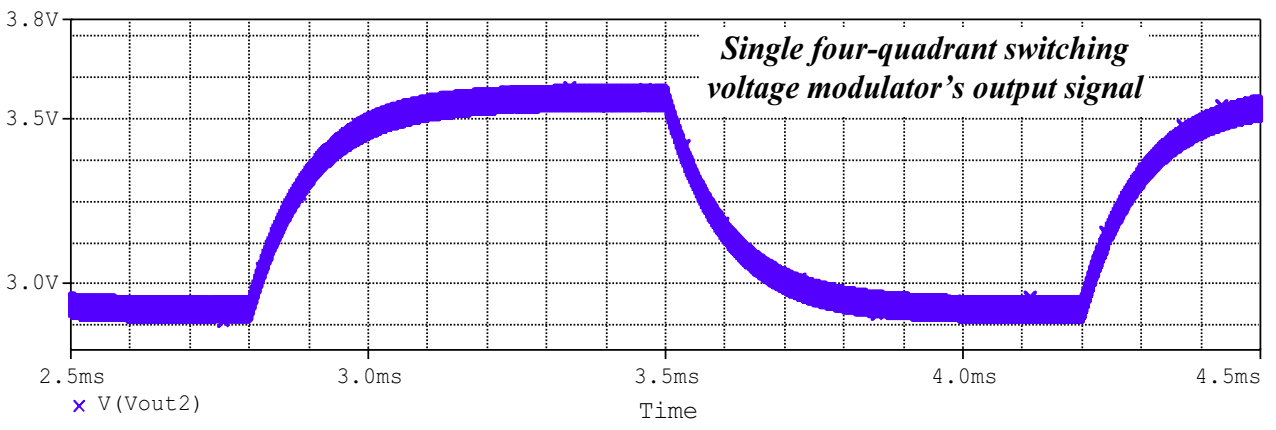

positive and negative values, the output voltage, $V_{\text {out }}$, will also follow the first one thanks to the four quadrants capability of the topology. However, notice that the current flowing through the linear regulator block will remain below $I_{\gamma}$, independently of the output voltage value, $V_{\text {out }}$. Therefore, the whole four-quadrant DC/DC linear-assisted switching regulator will have almost the same efficiency that the four-quadrant DC/DC switching converter.

\section{Results}

The discussed four-quadrant DC/DC linear-assisted switching regulator is designed and simulated using a discrete implementation as a proof of concept in order to explore and characterize the validity of the proposal. On the one hand, low frequency corroboration has been carried out in order to validate the four-quadrant linear-assisted DC/DC voltage regulator as a candidate for audio class-AD power amplifiers. Moreover, the response of the proposal is compared with the single four-quadrant switching voltage regulator. In this case, Fig. 5 shows the transient response to a single $20-\mathrm{kHz}$ tone of the linear-assisted switching regulator that provides a peak current of 4.0 A to the $1-\Omega$ output load (adjusting $V_{Z}=4 V_{\text {peak }}$ ). The values of the switching converter passive components are $L_{1}=10 \mu \mathrm{H}$, $L_{2}=100 \mu \mathrm{H}, C_{1}=100 \mu \mathrm{F}$, and input voltage $V_{\text {in }}=5 \mathrm{~V}$. On the other hand, adjusting $R_{m}=1 \Omega$, and $V_{\text {ref }}=0.2 \mathrm{~V}$, we have $I_{\gamma}=200 \mathrm{~mA}$. Notice the achieved tracking of the output voltage (or current) and $V_{Z}$. It is important to highlight that the output current (and, thus, output voltage) is free of output ripples, and almost all the output is provided by the witching block. In fact, the linear block only provides a small percentage, with average value equal to $200 \mathrm{~mA}$.

In order to corroborate the efficiency of the proposed four-quadrant linear-assisted regulator in low frequency, Fig. 6 shows the efficiency of the circuit compared to its single four-quadrant switching and linear version counterparts. Note that efficiencies in the linear-assisted and switching versions are very similar, especially in full-load conditions. In particular, the maximum efficiency for the linear-assisted regulator is $86 \%$ when the maximum 
Fig. 9 Input and output signals of proposed four-quadrant linear-assisted voltage modulator, and the single fourquadrant switching voltage modulator with a multi-tone time-varying modulation input signal as a candidate for nonconstant envelope WCDMA
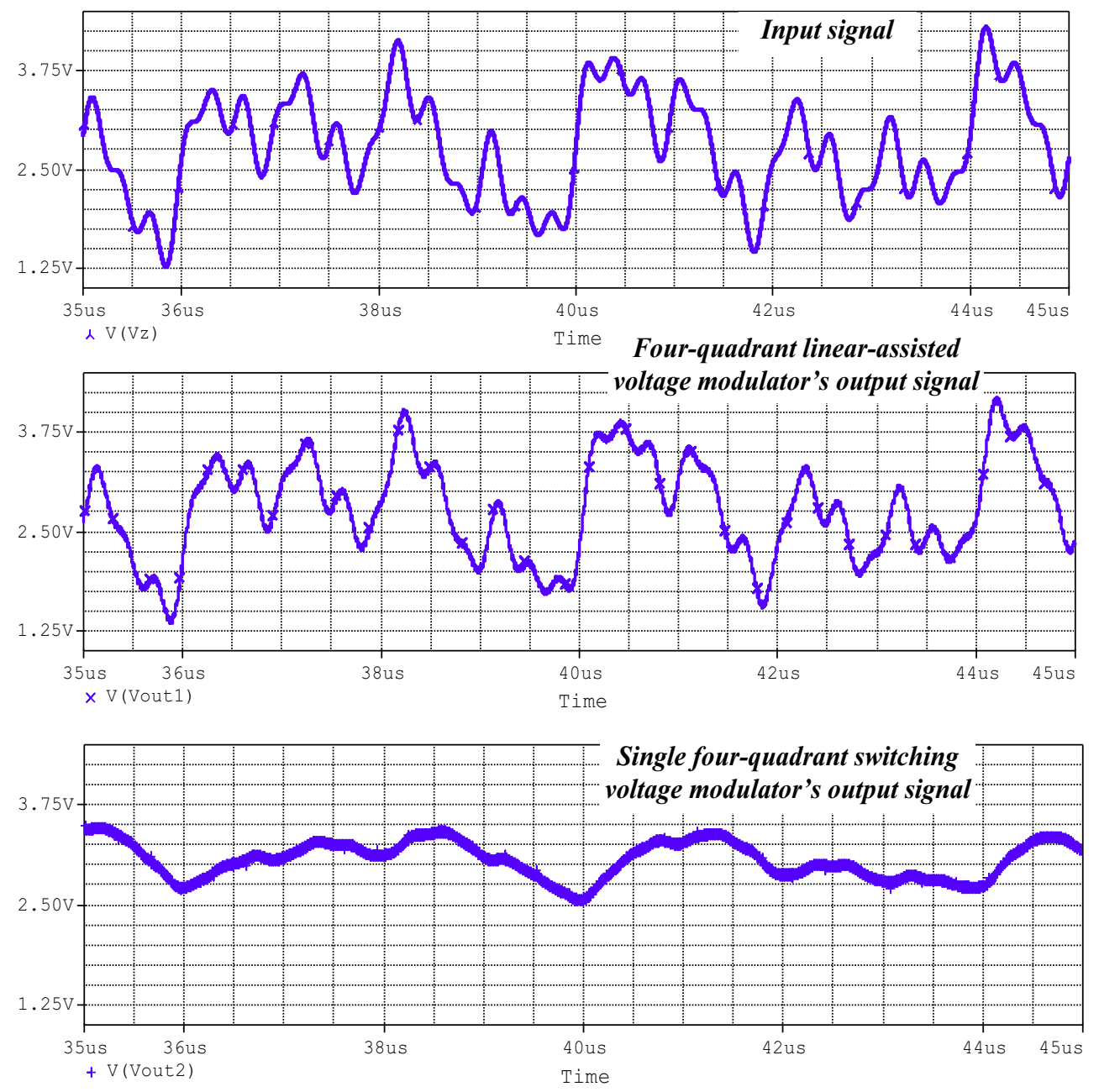

efficiency for the switching converter is slightly higher (around $87 \%$ ).

In addition, in order to evaluate the non-sinusoidal tracking capability of the proposed four-quadrant linearassisted voltage regulator for audio applications, a multitone signal, with 16 logarithmic distributed frequency components in the range of $20 \mathrm{~Hz}$ to $22 \mathrm{kHz}$, is applied to the circuit (as a candidate for class-AD power amplifier). This multi-tone signal is applied, on the one hand, to the proposed circuit. In addition, it is applied as time-varying modulation signal to produce the time-varying PWM and excite the switches of a single four-quadrant switching voltage regulator (with the same component values used in the linear-assisted proposal). The cut-off frequency of the converter for this signal is set to $200 \mathrm{kHz}$, and the switching frequencies $f_{s}$ is $1 \mathrm{MHz}$. In particular, Fig. 7 illustrates the time domain input and output signals. As it can be observed, the proposed four-quadrant linear-assisted voltage regulator operation is well, and it can track without important output ripples. However, the single four-quadrant switching regulator can track the input signal, but in a worst way than the proposal, and presenting significant ripple.

On the other hand, in order to validate the response of the four-quadrant linear-assisted proposal at high frequencies as a candidate topology for integrated envelope tracking applications in power supply of RFPAs, simulations have been carried out, and transient responses are studied. It allows comparing both the proposed four-quadrant linearassisted voltage regulator and the single four-quadrant switching voltage regulator at high frequencies. In particular, in this new case, circuit values of the proposed four-quadrant linear-assisted voltage regulator has been chosen in order to optimize the high-frequency response with $L_{1}=1 \mu \mathrm{H}$, $L_{2}=10 \mu \mathrm{H}, C_{1}=100 \mathrm{nF}$, and $V_{i n}=5 \mathrm{~V}$. Moreover, adjusting $R_{m}=1 \Omega$, and $V_{\text {ref }}=0.2 \mathrm{~V}, I_{\gamma}$ remains $200 \mathrm{~mA}$. Firstly, in order to verify the dynamic response capabilities of the proposed converter, transient response for CDMA IS95 specifications (transmit power is adjusted by $1 \mathrm{~dB}$ every $1.2 \mathrm{~ms}$, as requested by the base station $[12,13])$ is considered. In this case, a step stimulus was applied to the converters' control in order to change the PAs supply from 
Fig. 10 Comparison of average efficiencies of the four-quadrant switching modulator (blue line), the proposed four-quadrant linear-assisted regulator (red line), and linear modulator (pink line), with respect to variation in the output current (Color figure online)

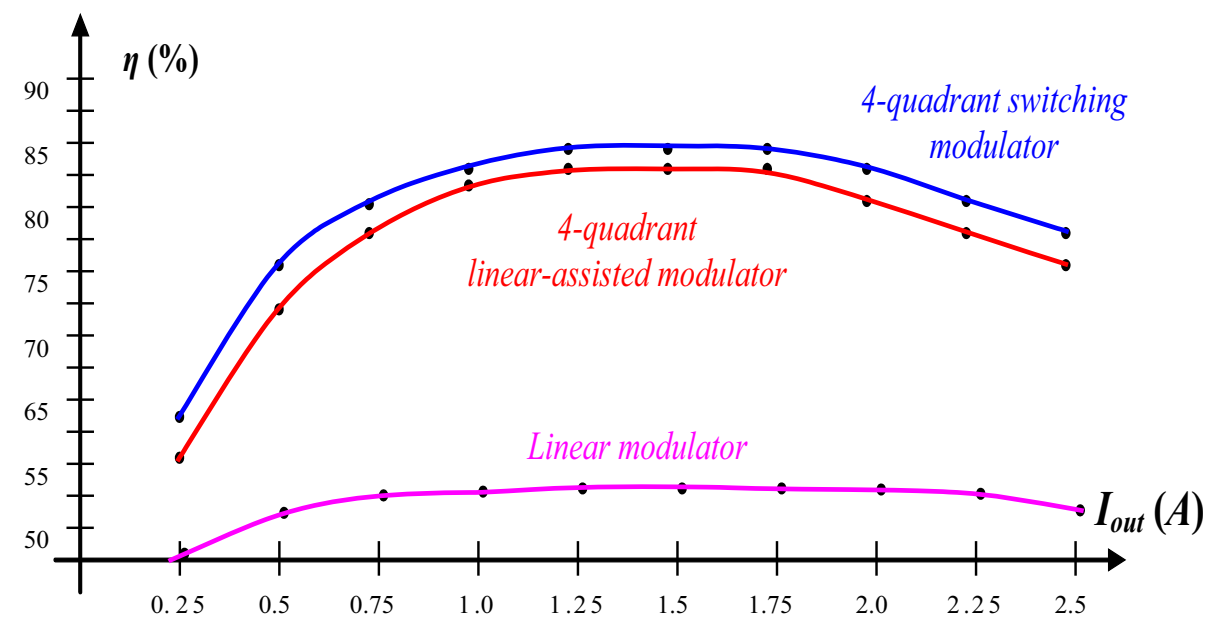

$2.95 \mathrm{~V}$ (output power of $22.4 \mathrm{dBm}$ ) to $3.6 \mathrm{~V}$ (output power of $24.1 \mathrm{dBm}$ ). Figure 8 shows these results; accordingly, it is seen that, in the worst case, the proposed four-quadrant linear-assisted voltage regulator responds to the power adjustment within $40 \mu \mathrm{s}$, whereas the single four-quadrant switching voltage regulator responds within $500 \mu \mathrm{s}$.

In addition, in order to evaluate the non-constant envelope tracking capability of the proposed four-quadrant linear-assisted voltage regulator, a multi-tone signal, in this case with 32 logarithmic distributed frequency components in the range of $100 \mathrm{~Hz}$ to $3.2 \mathrm{MHz}$ (as a candidate for WCDMA) has been considered. This multi-tone signal is applied, on the one hand, to the proposed circuit, and, in addition, as a time-varying modulation signal to produce the time-varying PWM and excite the switches of a single four-quadrant switching voltage regulator (with the same component values that used in the linear-assisted proposal). In this case, the cut-off frequency of the converter for this signal is set to $10 \mathrm{MHz}$, and the switching frequencies $f_{s}$ is $40 \mathrm{MHz}$. It is noted that the relation between the input signal bandwidth, the cut-off frequency, and the switching frequency for a converter in envelope elimination and restoration (EER) applications is addressed in [14]. In particular, Fig. 9 illustrates the time domain input and output signals. As it can be seen, the single four-quadrant switching regulator barely can track the input signal, also presenting significant ripple. However, the proposed fourquadrant linear-assisted voltage regulator operation is well, and it can track without significant ripples.

Finally, in order to corroborate the efficiency of the proposed four-quadrant linear-assisted modulator for this particular application in WCDMA, Fig. 10 shows the average efficiency of the circuit compared to the single four-quadrant switching block and the linear amplifier block. Note that the efficiencies in both cases are very similar, especially in medium-load conditions. In particular, the maximum efficiency for the linear-assisted regulator is $82 \%$ when the maximum efficiency for the switching converter is slightly higher (around $84 \%$ ).

\section{Conclusions}

In this Mixed Signal Letter, a new design of a four-quadrant DC/DC voltage regulator based on linear-assisted structure has been presented. In this proposal, a class-AB linear voltage regulator assists a four-quadrant switching DC/DC converter that uses only two switches, simplifying the architecture and obtaining a compact circuit with advantages of both alternatives; i.e., high efficiency, low output ripple, and fast reaction to the load changes.

In order to reduce the power dissipation in the linear regulator, it is considered as an assisting circuit for providing just a small fraction of the load current. The circuit can be focused on obtaining good-performance power-supply modulators for envelope tracking techniques in power amplifiers. Finally, this four-quadrant proposal can be a candidate not only in the power supply path of the supply modulator, but also in the signal path (with a suitable component design according to the frequency requirements) in order to overcome the well-known drawbacks associated to audio and RF class-D power amplifiers.

Acknowledgments This work has been partially supported by the Spanish Ministerio de Economía y Competitividad by project DPI2013-47799-C2-2-R.

\section{References}

1. Bachir, S., Calinoiu, N. E., \& Duvanaud, C. (2015). New RF power amplifiers modeling and identification for wideband applications. Analog Integrated Circuits and Signal Processing, 83, 161-172.

2. Egolf, E. (2007). Intelligent power management: a method to improve 2G/3G handset talk time. Microwave Journal, 50(7), 94-100. 
3. Villar, G., Alarcón, E., Guinjoan, F., \& Poveda, A. (2005). Efficiency-oriented switching frequency tuning for a buck switching power converter. IEEE International Symposium on Circuits and Systems (Vol. 3, pp. 2473-2476). Kobe, Japan.

4. Turkson, R., Prakash, S., Silva-Martínez, J., \& Martínez-García, H. (2013). Envelope tracking technique with bang-bang slew-rate enhancer for linear wideband RF PAs. In Proceedings of the 2013 IEEE $56^{\text {th }}$ International Midwest Symposium on Circuits and Systems (pp. 629-632). Columbus, OH, USA.

5. Feipeng, W., Kimball, D. F., Lie, D. Y., Asbeck, P. M., \& Larson, L. E. (2007). A monolithic high-efficiency 2.4-GHz 20-dBm SiGe BiCMOS envelope-tracking OFDM power amplifier. IEEE Journal of Solid-State Circuits, 42(6), 1271-1281.

6. Martínez-García, H. (2015). Design of a constant switching frequency control-based linear-Assisted DC/DC regulator for photovoltaic solar-powered facilities. In Proceedings of the 2015 IEEE $6^{\text {th }}$ International Symposium on Power Electronics for Distributed Generation Systems (pp. 1-5). Aachen, Germany.

7. Pillonnet, G., Cellier, R., Nagari, A., Lombard, P., \& Abouchi, N. (2013). Sliding mode audio class-D amplifier for portable devices. Analog Integrated Circuits and Signal Processing, 74, 439-451.

8. Hwang, Y.-S., Shen, J.-H., Chen, J.-J., \& Fan, M.-R. (2013). Performance comparison of integrated fully-differential filterless class-D amplifiers with different feedback techniques. Analog Integrated Circuits and Signal Processing, 76, 167-177.

9. Davide, C., Malcovati, P., Crespi, L., Lee, K., \& Baschirotto, A. (2014). A design methodology for high-order class-D audio amplifiers. Analog Integrated Circuits and Signal Processing, 78, 785-798.

10. Wu, A. (2014). Four-quadrant DC/DC switching regulator smoothly transitions from positive to negative output voltages for FPGA. EDN Europe, March 2014, p. 20.

11. Martínez, H., \& Conesa, A. (2007). Modeling of linear-assisted DC-DC converters. In Proceedings of the $200718^{\text {th }}$ European Conference on Circuit Theory and Design (pp. 611-614). Seville, Spain.

12. Sahu, B., \& Rincón-Mora, G. A. (2004). A high-efficiency linear RF power amplifier with a power-tracking dynamically adaptive buck-boost supply. IEEE Transactions on Microwave Theory and Techniques, 52(1), 112-120.

13. Harte, L., Levine, R., \& Kikta, R. (2002). $3 G$ wireless demystified. NewYork: McGraw-Hill.

14. Marco, L., Alarcón, E., \& Maksimovic, D. (2006). Effects of switching power converter nonidealities in envelope elimination and restoration technique. IEEE International Symposium on Circuits and Systems (ISCAS'06). pp. 3137-3140. Island of Kos, Greece.

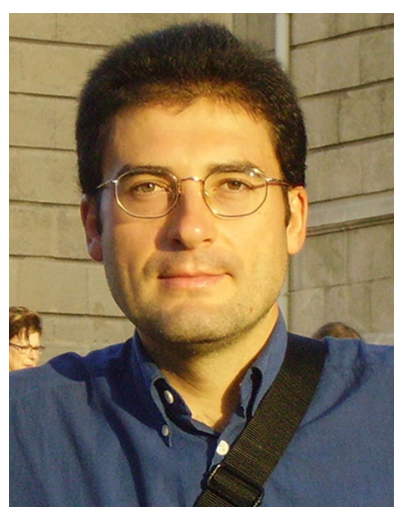

Herminio Martínez-García received the B.E. degree (National Award) in Electrical Engineering, the M.S. degree (National Award) in Electronics Engineering and the Ph.D. degrees in Electronics Engineering (all three with honors) from the Technical University of Catalonia (UPC, BarcelonaTech) in Barcelona, Spain, in 1994, 1998 and 2003, respectively. During the period 1995-1998, Dr. Martinez-Garcia was a half-time Assistant Professor at the Department of Electronics of the College of Industrial Engineering of Barcelona (EUETIB-CEIB), where he became a full- time Assistant Professor at the same department in September 1998. In September 2000 he joined the Department of Electronics Engineering of the UPC-BarcelonaTech, where he became an Associate Professor in 2006 and researcher with the Energy Processing and Integrated Circuits (EPIC) Group of the UPC. From October 2008 to March 2009, he was a Visiting Professor at the Analog \& Mixed Signal Center (AMSC) of the Department of Electrical and Computer Engineering of the Texas A\&M University (TAMU) at College Station, TX (USA). Professor Martinez-Garcia currently teaches analog circuits design, communication systems, and data acquisition and control systems. His research focuses on the area of DC-DC power converters and their control, and analog circuit design with emphasis in analog microelectronics and particular interest in continuous-time filters and automating tuning design, analog and energy processing circuits for energy harvesting. He has participated in five Spanish national research projects. He has authored or co-authored about sixty scientific papers in journals and conference proceedings and 15 books and book chapters. Dr. Martinez is a member of the IEEE Solid-State Circuits, Power Electronics, and Education Societies. He has been organizing committee member and technical program committee (TPC) member of different IEEE Conferences such as ETFA 2014 and COMPENG 2014. In addition, he has served as a reviewer for different Journals, Transactions, and Conferences such as Electronics Letters, Analog Integrated Circuits and Signal Processing, Elsevier Integration, the VLSI Journal, and also ISCAS, MWSCAS, ICECS, LASCAS, ECCTD, ETFA, etc.

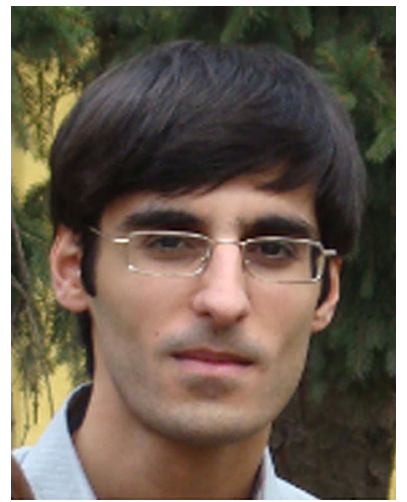

Alireza Saberkari received the B.Sc. degree in Electrical Engineering from Iran University of Science and Technology (IUST), Tehran/University of Guilan, Rasht, Iran, in 2002 and the M.Sc. and Ph.D. degrees both in Electrical Engineering from Iran University of Science and Technology (IUST), Tehran, Iran, in 2004 and 2010, respectively (all with honors). Since 2010, he has been with the Department of Electrical Engineering at University of Guilan as an Assistant Professor. During the period 2008-2009, he joint the group of Energy Processing Integrated Circuits (EPIC), Department of Electronics Engineering, Technical University of Catalunya (UPC), Barcelona, Spain, as a Visiting Scholar and worked on "CMOS Linear Low-Dropout Regulators for Wideband-Tracking Linear-Assisted Scheme" \& "RF Transmitter Architectures Considering Wideband Adaptive Supply of RF PA". He has authored or coauthored more than 50 international scientific publications including journals and conference proceedings. He was the technical program committee (TPC) member of the IEEE Latin American Symposium on Circuits and Systems (LASCAS'13, LASCAS'14, and LASCAS'15) and IEEE International Conference on Emerging Technologies and Factory Automation (ETFA'14), and also the organizing committee member of the IEEE International Conference on Pattern Recognition and Image Analysis (IPRIA'15). He has served as a reviewer for the IEEE Transactions on Electron Devices, Electronics Letters, Analog Integrated Circuits and Signal Processing, Wiley International Journal of Circuit Theory and Applications, International Journal of Electronics, Elsevier Microelectronics Journal, Elsevier integration, the VLSI Journal, Journal of Circuits, Systems, and Computers, Electronics and Electrical Engineering, International Journal for the Computation and Mathematics in Electrical and Electronic Engineering, Journal of Low Power Electronics, International Journal of Signal and Data Processing, Iranian Journal of 
Electrical and Computer Engineering, and also ISCAS, MWSCAS, ICECS, LASCAS, ECCTD, ETFA, and ISWTA conferences. His fields of interest include the areas of Analog, RF, and Mixed-Signal Microelectronics with particular interest in On-Chip Power Management Circuits, Analog Circuits for Energy Harvesting
Applications and Biomedical Implants, Linear and Low-Dropout Regulators, Current-Mode Circuit Design, CMOS LNAs and Mixers, RF Power Amplifiers, and Low-Power and Low-Voltage Integrated Circuits. Dr. Saberkari is a member of IEEE Solid-State Circuits and Circuits and Systems societies. 